\title{
EULER-LAGRANGE RADICAL FUNCTIONAL EQUATIONS WITH SOLUTION AND STABILITY
}

\author{
MURALI RAMDOSS, DIVYAKUMARI PACHAIYAPPAN, AND HEMEN DUTTA \\ Received 08 October, 2019
}

\begin{abstract}
In this article, we introduce the generalized Euler-Lagrange radical functional equations of type sextic and quintic. Also, we obtain their general solution and investigate the generalized Hyers-Ulam-Rassias stability in modular spaces using fixed point concept with suitable counter examples.
\end{abstract}

2010 Mathematics Subject Classification: 39B52; 39B72; 39B82; 46B03

Keywords: Modular space, quintic and sextic functioanl equations, Hyers- Ulam- Rassias stability, fixed point theorem

\section{INTRODUCTION}

Ulam [13] raised the infamous stability problem of functional equations in 1940 at the University of Wisconsin. The solution for the Ulam problem garnered world wide attention and finally came to be identified as generalized Hyers-Ulam, generalized Hyers-Ulam-Rassias, Ulam-Găvruta-Rassias and JMR stabilities of functional equations. One can refer $([1,7,8,10-12])$.

In the probabilistic normed spaces, Mohammad Bagher Ghaemi et al. [6] analyzed the stability for the sextic and quintic mappings.

In the quasi- $\beta$-normed spaces via fixed point method, Tian Zhou Xu et al. [15] introduced the following functional equation of quintic type

$$
\begin{gathered}
g(m+3 n)-5 g(m+2 n)+10 g(m+n)-10 g(m) \\
+5 g(m-n)-g(m-2 n)=120 g(n)
\end{gathered}
$$

and sextic type

$$
\begin{gathered}
g(m+3 n)-6 g(m+2 n)+15 g(m+n)-20 g(m)+15 g(m-n) \\
-6 g(m-2 n)+g(m-3 n)=720 g(n)
\end{gathered}
$$

and also investigated their stabilities related to Ulam problem. 
In Felbin spaces, Pasupathi Narasimman et al. [9] introduced generalized sextic and quintic functional equations

$$
\begin{aligned}
& g(a m+n)+g(a m-n)+g(m+a n)+g(m-a n) \\
& =\left(a^{4}+a^{2}\right)[g(m+n)+g(m-n)]+2\left(a^{6}-a^{4}-a^{2}+1\right)[g(m)+g(n)] \\
& a[g(a m+n)+g(a m-n)]+g(m+a n)+g(m-a n) \\
& \quad=\left(a^{4}+a^{2}\right)[g(m+n)+g(m-n)]+2\left(a^{6}-a^{4}-a^{2}+1\right) g(m)
\end{aligned}
$$

with general solution and stability for $a \in \mathbb{R}-\{0, \pm 1\}$.

Using fixed point theory, Zamani Eskandani and John Michael Rassias [5], Kittipong Wongkum [14] are obtained modular stability of $\boldsymbol{\gamma}$-quartic and cubic functional equations.

In quasi- $\beta$-normed spaces, In Goo Cho et al. [3] analyzed the Ulam stability problem for the quintic functional equation of the form

$$
\begin{gathered}
2 g(2 m+n)+2 g(2 m-n)+g(m+2 n)+g(m-2 n) \\
=20[g(m+n)+g(m-n)]+90 g(m) .
\end{gathered}
$$

In 2015, Abasalt Bodaghi et al.[2] analyzed the general solution and stability of a mixed type of quintic-additive functional equation of the form

$$
\begin{aligned}
& g(3 m+n)-5 g(2 m+n)+g(2 m-n)+10 g(m+n)-5 g(m-n) \\
& \quad=10 g(n)+4 g(2 m)-8 g(m)
\end{aligned}
$$

in real numbers.

Motivated from the above investigations on sextic and quintic functional equations, in this paper we introduce the following new generalized Euler-Lagrange radical quintic and sextic functional equations

$$
\begin{gathered}
f(a x+y)+f(a x-y)+f(x+a y)+f(x-a y) \\
=\left(a+a^{2}\right)\{f(x+y)+f(x-y)\}-2\left(a+a^{2}-a^{5}-1\right) f(x) \\
+10\left(a^{4}-a^{2}\right) f\left(\sqrt[5]{x y^{4}}\right)+20\left(a^{3}-a\right) f\left(\sqrt[5]{x^{3} y^{2}}\right), \\
f(a x+y)+f(a x-y)+f(x+a y)+f(x-a y) \\
=\left(a+a^{2}\right)\{f(x+y)+f(x-y)\}-2\left(a+a^{2}-a^{6}-1\right)\{f(x)+f(y)\} \\
+30\left(a^{4}-a\right)\left\{f\left(\sqrt[6]{x^{4} y^{2}}\right)+f\left(\sqrt[6]{x^{2} y^{4}}\right)\right\},
\end{gathered}
$$

for a fixed real $a$ and $a \neq 0, \pm 1$. Mainly we obtain their general solution and investigate their stabilities related to Ulam problem in modular spaces. The definitions related to modular space and fixed point theory to establish our main theorem can be referred in [4].

The paper structured as follows: In Section-2, we obtain the general solution of the functional equations (1.1) and (1.2). In Section-3 and in Section 4, authors discuss 
generalized Hyers-Ulam-Rassias, Hyers-Ulam and Hyers-Ulam-Rassias stabilities of quintic and sextic functional equations respectively in modular spaces using fixed point theory. Finally the conclusion given in section-5.

2. General Solution of (1.1) And (1.2)

Theorem 1. If $f$ satisfies (1.1), then a mapping $f: X \rightarrow Y$ is quintic and odd.

Proof. Consider $f$ satisfies (1.1). Setting $(x, y)=(0,0)$ in $(1.1)$, we get $f(0)=0$. Replacing $(x, y)$ by $(x, 0)$ in $(1.1)$, we arrive

$$
f(a x)=a^{5} f(x)
$$

for all $x \in X$. Therefore $f$ is quintic. Setting $(x, y)$ by $(0, x)$ in (1.1) and using (2.1) leads

$$
f(-x)=-f(x)
$$

for all $x \in X$ and hence $f$ is odd.

Theorem 2. If $f$ satisfies (1.2), then a mapping $f: X \rightarrow Y$ is sextic and even.

Proof. Consider $f$ satisfies (1.2). Setting $(x, y)=(0,0)$ in (1.2), we get $f(0)=0$. Replacing $(x, y)$ by $(x, 0)$ in (1.2), we arrive

$$
f(a x)=a^{6} f(x)
$$

for all $x \in X$. Therefore $f$ is sextic. Setting $(x, y)$ by $(0, x)$ in (1.2) and using (2.3) leads

$$
f(-x)=f(x)
$$

for all $x \in X$ and hence $f$ is even.

\section{Stability of FUnCTIONAL EQUATion(1.1)}

In this section, we determine the generalized Hyers-Ulam stability concerning the generalized Euler-Lagrange radical quintic functional equation (1.1) in modular spaces by using fixed point theory.

For mapping $\rho: M \rightarrow X_{\xi}$, consider

$$
\begin{aligned}
& D_{q} f(x, y):=f(x+a y)+f(x-a y)+f(a x+y)+f(a x-y) \\
& \quad-\left(a+a^{2}\right)\{f(x+y)+f(x-y)\}+2\left(a+a^{2}-a^{5}-1\right) f(x) \\
& \quad-10\left(a^{4}-a^{2}\right) f\left(\sqrt[5]{x y^{4}}\right)-20\left(a^{3}-a\right) f\left(\sqrt[5]{x^{3} y^{2}}\right)
\end{aligned}
$$

for all $x, y \in M$ with $a \neq 0, \pm 1$

Theorem 3. Consider a mapping $\rho: M^{2} \rightarrow[0,+\infty)$ such that

$$
\lim _{n \rightarrow \infty} \frac{1}{a^{5 n}} a \rho\left\{a^{n} x, a^{n} y\right\}=0,
$$


and

$$
\rho\{a x, a y\} \leq a^{5} \psi \rho\{x, y\}, \forall x, y \in M,
$$

for $\psi<1$. If $f: M \rightarrow X_{\xi}$ fulfill the inequality

$$
\xi\left(D_{q} f(x, y)\right) \leq \rho(x, y),
$$

$\forall x, y \in M$. Then $Q_{a}: M \rightarrow X_{\xi}$ a unique quintic mapping exists, such that

$$
\xi\left(Q_{a}(x)-f(x)\right) \leq \frac{1}{2 a^{5}(1-\psi)} \rho(x, 0), \forall x \in M .
$$

Where $M$ is linear space and $X_{\xi}$ is modular space which is complete with Fatou property.

Proof. Consider $N=\xi^{\prime}$ and define $\xi^{\prime}$ on $N$ as,

$$
\xi^{\prime}(q)=: \inf \{a>0: \xi(f(y)) \leq a \rho(x, 0), \forall x \in M\} .
$$

One can easily prove $\xi^{\prime}$ is convex modular with Fatou property on $N$ and $N_{\xi^{\prime}}$ is $\xi$-complete, see [5]. Consider the function $\sigma: N_{\xi^{\prime}} \rightarrow N_{\xi^{\prime}}$ defined by

$$
\sigma f(x)=\frac{1}{a^{5}} f(a x)
$$

for all $x \in M$ and $a \in N_{\xi^{\prime}}$. Let $p, r \in N_{\xi^{\prime}}$ and $a \in[0,1]$ with $\xi^{\prime}(p-r)<a$. By definition of $\xi^{\prime}$, we get

$$
\xi(p(x)-r(x)) \leq a \rho(x, 0)
$$

for all $x \in M$. By (3.2) and (3.6), we obtain

$$
\xi\left(\frac{p(a x)}{a^{5}}-\frac{r(a x)}{a^{5}}\right) \leq \frac{1}{a^{5}} \xi(p(a x)-r(a x)) \leq \frac{1}{a^{5}} a \rho(a x, 0) \leq a \psi \rho(x, 0),
$$

for all $x \in M$. Hence, $\sigma$ is a $\xi^{\prime}-$ contraction. From (3.3), we obtain

$$
\xi\left(\frac{f(a x)}{a^{5}}-f(x)\right) \leq \frac{1}{2 a^{5}} \rho(x, 0),
$$

for all $x \in M$. Substituting $x$ by $a x$ in (3.7), we get

$$
\xi\left(\frac{f\left(a^{2} x\right)}{a^{5}}-f(a x)\right) \leq \frac{\rho(a x, 0)}{2 a^{5}}, \forall x \in M .
$$

We obtain from (3.7) and (3.8) that

$$
\begin{aligned}
& \xi\left(\frac{f\left(a^{2} x\right)}{a^{10}}-f(x)\right) \\
& \leq \xi\left(\frac{f\left(a^{2} x\right)}{a^{10}}-\frac{f(a x)}{a^{5}}\right)+\xi\left(\frac{f(a x)}{a^{5}}-f(x)\right) \\
& \leq \frac{1}{2 a^{10}} \rho(a x, 0)+\frac{1}{2 a^{5}} \rho(x, 0), \forall x \in M .
\end{aligned}
$$


We get by induction,

$$
\begin{aligned}
\xi\left(\frac{f\left(a^{n} x\right)}{a^{5 n}}-f(x)\right) & \leq \sum_{i=1}^{n} \frac{1}{2 a^{5 i}} \rho\left(a^{i-1} x, 0\right) \\
& \leq \frac{1}{2 \psi a^{5}} \rho(x, 0) \sum_{i=1}^{n} \psi^{i} \\
& \leq \frac{1}{2 a^{5}(1-\psi)} \rho(x, 0), \forall x \in M .
\end{aligned}
$$

We obtain from (3.10),

$$
\begin{aligned}
\xi\left(\frac{f\left(a^{n} x\right)}{a^{5 n}}-\frac{f\left(a^{s} x\right)}{a^{5 s}}\right) & \leq \frac{1}{2} \xi\left(2 \frac{f\left(a^{n} x\right)}{a^{5 n}}-2 f(x)\right)+\frac{1}{2} \xi\left(2 \frac{f\left(a^{5} x\right)}{a^{5 s}}-2 f(x)\right) \\
\leq & \frac{\kappa}{2} \xi\left(\frac{f\left(a^{n} x\right)}{a^{5 n}}-f(x)\right)+\frac{\kappa}{2} \xi\left(\frac{f\left(a^{5} x\right)}{a^{5 s}}-f(x)\right) \\
\leq & \frac{\kappa}{2 a^{5}(1-\psi)} \rho(x, 0), \forall x \in M
\end{aligned}
$$

where $n, s \in \mathfrak{N}$. Thus

$$
\xi^{\prime}\left(\sigma^{n} f-\sigma^{s} f\right) \leq \frac{\kappa}{2 a^{5}(1-\psi)},
$$

hence the boundedness exists of an orbit of $\sigma$ at $f .\left\{\tau^{n} f\right\}$ is $\xi^{\prime}$-converges to $Q_{a} \in N_{\xi^{\prime}}$ by Theorem 1.5 in [5]. By $\xi^{\prime}$-contractivity of $\sigma$, we get

$$
\xi^{\prime}\left(\sigma^{n} f-\sigma Q_{a}\right) \leq \psi \xi^{\prime}\left(\sigma^{n-1} f-Q_{a}\right) .
$$

Allowing $n \rightarrow \infty$ and by Fatou property of $\xi^{\prime}$, we get

$$
\begin{aligned}
\xi^{\prime}\left(\sigma Q_{a}-Q_{a}\right) & \leq \liminf _{n \rightarrow \infty} \xi^{\prime}\left(\sigma Q_{a}-\sigma^{n} f\right) \\
& \leq \psi \liminf _{n \rightarrow \infty} \xi^{\prime}\left(Q_{a}-\sigma^{n-1} f\right)=0 .
\end{aligned}
$$

Hence, $Q_{a}$ is a fixed point of $\sigma$. In (3.3), changing $(x, y)$ by $\left(a^{n} x, a^{n} y\right)$, we obtain

$$
\xi\left(\frac{1}{a^{5 n}} D_{a} f\left(a^{n} x, a^{n} y\right)\right) \leq \frac{1}{a^{5 n}} \rho\left(a^{n} x, a^{n} y\right), \forall x, y \in M .
$$

By Theorem 1 and allowing $n \rightarrow \infty, Q_{a}$ is quintic and using (3.10), we arrive (3.4). For the uniqueness of $Q_{a}$, consider another quintic mapping $Q: M \rightarrow X_{\xi}$ satisfying (3.4). So that, $Q$ is fixed point of $\sigma$.

$$
\xi^{\prime}\left(Q_{a}-Q\right)=\xi^{\prime}\left(\sigma Q_{a}-\sigma Q\right) \leq \psi \xi^{\prime}\left(Q_{a}-Q\right) .
$$

From (3.13), we get $Q_{a}=Q$. Hence the proof.

Proof of following Corollaries 1 and 2 follows that, all normed space implies modular space of modular $\xi(x)=\|x\|$. 
Corollary 1. Assume $\rho$ is a mapping from $M^{2}$ to $[0,+\infty)$ for

$$
\lim _{n \rightarrow \infty} \frac{1}{a^{5 n}} \rho\left\{a^{n} x, a^{n} y\right\}=0
$$

and

$$
\rho\{a x, a y\} \leq a^{5} \psi \rho\{x, y\}, \forall x, y \in M, \psi<1 .
$$

If $f: M \rightarrow X$ satisfies the condition for $X$ is Banach space

$$
\left\|D_{q} f(x, y)\right\| \leq \rho(x, y),
$$

$\forall x, y \in M$. Then a unique $Q_{a}: M \rightarrow X$ quintic mapping exists, hence

$$
\left\|Q_{a}(x)-f(x)\right\| \leq \frac{\rho(x, 0)}{2 a^{5}(1-\psi)}
$$

for all $x \in M$.

Theorem 4. Assume that,

$$
\lim _{n \rightarrow \infty} \kappa^{5 n} \rho\left(\frac{x}{a^{n}}, \frac{y}{a^{n}}\right)=0,
$$

where $\rho$ is a mapping from $M^{2}$ to $[0,+\infty)$ and

$$
\rho\left(\frac{x}{a}, \frac{y}{a}\right) \leq \frac{\psi}{2 a^{5}} \rho\{x, y\}, \forall x, y \in M, \psi<1 .
$$

If $f: M \rightarrow X_{\xi}$ fulfills the inequality

$$
\xi\left(D_{q} f(x, y)\right) \leq \rho(x, y),
$$

$\forall x, y \in M$. Then a unique $Q_{a}: M \rightarrow X_{\xi}$ quintic mapping exists, such that

$$
\xi\left(Q_{a}(x)-f(x)\right) \leq \frac{\psi}{2 a^{5}(1-\psi)} \rho(x, 0), \forall x \in M .
$$

Proof. Considering $x$ by $\frac{x}{a}$ in (3.5) of Theorem 3 and proceeding similar to that of Theorem 3, we complete the proof.

Corollary 2. Assume that,

$$
\lim _{n \rightarrow \infty} \sigma^{5 n} \rho\left(\frac{x}{a^{n}}, \frac{y}{a^{n}}\right)=0,
$$

where $\rho$ is a mapping from $M^{2}$ to $[0,+\infty)$ and

$$
\rho\left(\frac{x}{a}, \frac{y}{a}\right) \leq \frac{\psi}{a^{5}} \rho\{x, y\}, \forall x, y \in M, \psi<1 .
$$

If $f: M \rightarrow X$ fulfills the inequality

$$
\left\|D_{q} f(x, y)\right\| \leq \rho(x, y),
$$

$\forall x, y \in M$. Then a unique $Q_{a}: M \rightarrow X$ quintic mapping exists, such that

$$
\left\|Q_{a}(x)-f(x)\right\| \leq \frac{\psi}{2 a^{5}(1-\psi)} \rho(x, 0), \forall x \in M .
$$


Using Corollaries 1 and 2, the Hyers-Ulam and generalized Hyers-Ulam stabilities of (1.1) are obtain in the following corollaries.

Corollary 3. Assume $\rho$ is a mapping from $M^{2}$ to $[0,+\infty), X$ be a Banach space and $\varepsilon \geq 0$ be a real number such that

$$
\lim _{n \rightarrow \infty} \frac{1}{a^{5 n}} \rho\left\{a^{n} x, a^{n} y\right\}=0
$$

and

$$
\rho\{a x, a y\} \leq a^{5} \psi \rho\{x, y\}, \forall x, y \in M, \psi<1 .
$$

If $f: M \rightarrow X$ fulfills

$$
\left\|D_{q} f(x, y)\right\| \leq \varepsilon,
$$

$\forall x, y \in M$. Then a unique $Q_{a}: M \rightarrow X$ quintic mapping exists and defined by $Q_{a}(x)=$ $\lim _{n \rightarrow \infty} \frac{f\left(a^{n} x\right)}{a^{5 n}}$ so that

$$
\left\|Q_{a}(x)-f(x)\right\| \leq \frac{\varepsilon}{2\left(a^{5}-1\right)},
$$

for all $x \in M$ and $a \neq 0, \pm 1$.

Corollary 4. If $f: M \rightarrow X$ fulfills the inequality for $M$ and $X$ are linear space and Banach space, respectively.

$$
\left\|D_{q} f(x, y)\right\| \leq \varepsilon\left(\|x\|^{p}+\|y\|^{q}\right),
$$

$\forall x, y \in M$ with $0 \leq p, q<5$ or $p, q>5$ for some $\varepsilon \geq 0$. Then a unique quintic mapping $Q_{a}: M \rightarrow X$ exists and defined by $Q_{a}(x)=\lim _{n \rightarrow \infty} \frac{f\left(a^{n} x\right)}{a^{5 n}}$, so that

$$
\left\|Q_{a}(x)-f(x)\right\| \leq \frac{\varepsilon}{\left|2\left(a^{5}-a^{p}\right)\right|}\|x\|^{p}, \forall x \in M, a \neq 0, \pm 1 .
$$

For $p=5$ in Corollary 4, we will provide an counter example to prove that the functional equation (1.1) is not stable.

Example 1. If $\psi: M \rightarrow X$ fulfills the inequality for $M$ and $X$ are linear space and Banach space, respectively. Let $k \geq 0$ be a real number such that

$$
\psi(x)= \begin{cases}k x^{5}, & \text { if }|x|<1, \\ k, & \text { otherwise }\end{cases}
$$

and a function $f: M \rightarrow X$ is defined by

$$
f(x)=\sum_{s=0}^{\infty} \frac{\varphi\left(a^{s} x\right)}{\left(a^{5}\right)^{s}}
$$

for all $x \in M$. Then $f$ satisfies the functional inequality

$$
f(a x+y)+f(a x-y)+f(x+a y)+f(x-a y)
$$




$$
\begin{aligned}
& -\left(a+a^{2}\right)\{f(x+y)+f(x-y)\}+2\left(a+a^{2}-a^{5}-1\right) f(x) \\
& -10\left(a^{4}-a^{2}\right) f\left(\sqrt[5]{x y^{4}}\right)-20\left(a^{3}-a\right) f\left(\sqrt[5]{x^{3} y^{2}}\right) \mid \\
& \leq\left(\frac{2 a^{10}-16 a^{11}-6 a^{12}+20 a^{13}+10 a^{14}-2 a^{15}}{a^{5}-1}\right) k\left(|x|^{5}+|y|^{5}\right)
\end{aligned}
$$

for all $x, y \in M$. Then there do not exist a quintic mapping $Q: M \rightarrow X$ and a constant $\alpha>0$ such that

$$
|f(x)-Q(x)| \leq \alpha|x|^{5} \text { for all } x \in M
$$

Proof. Now

$$
|f(x)| \leq \sum_{s=0}^{\infty} \frac{\left|\psi\left(a^{s} x\right)\right|}{\left|a^{5 s}\right|}=\sum_{s=0}^{\infty} \frac{k}{a^{5 s}}=\frac{a^{5} k}{a^{5}-1} .
$$

Therefore we see that $f$ is bounded. We are going to prove that $f$ satisfies (3.32).

If $x=y=0$, then (3.32) is trivial. If $|x|^{5}+|y|^{5} \geq \frac{1}{a^{5}}$, then the left-hand side of (3.32) is less than $\left(\frac{2 a^{5}-16 a^{6}-6 a^{7}+20 a^{8}+10 a^{9}-2 a^{10}}{n^{5}-1} k\right)$. Now suppose that $0<|x|^{5}+|y|^{5}<\frac{1}{a^{5}}$. Then there exists a positive integer $t$ such that

$$
\frac{1}{\left(a^{5}\right)^{t+1}} \leq|x|^{5}+|y|^{5}<\frac{1}{\left(a^{5}\right)^{t}}
$$

so that

$$
\left(a^{5}\right)^{t-1} x^{5}<\frac{1}{a^{5}},\left(a^{5}\right)^{t-1} y^{5}<\frac{1}{a^{5}}
$$

and, consequently,

$$
\begin{array}{r}
a^{t-1}(x), a^{t-1}(a x+y), a^{t-1}(a x-y), a^{t-1}(x+a y), a^{t-1}(x-a y), a^{t-1}(x+y), \\
a^{t-1}(x-y), a^{t-1}\left(\sqrt[5]{x y^{4}}\right), a^{t-1}\left(\sqrt[5]{x^{3} y^{2}}\right) \in(-1,1) .
\end{array}
$$

Therefore for each $s=0,1, \ldots, t-1$, we have

$$
\begin{array}{r}
a^{s}(x), a^{s}(a x+y), a^{s}(a x-y), a^{s}(x+a y), a^{s}(x-a y), a^{s}(x+y), \\
a^{s}(x-y), a^{s}\left(\sqrt[5]{x y^{4}}\right), a^{s}\left(\sqrt[5]{x^{3} y^{2}}\right) \in(-1,1) .
\end{array}
$$

and

$$
\begin{aligned}
& \psi\left(a^{s}(a x+y)\right)+\psi\left(a^{s}(a x-y)\right)+\psi\left(a^{s}(x+a y)\right)+\psi\left(a^{s}(x-a y)\right) \\
& \quad-\left(a+a^{2}\right)\left\{\psi\left(a^{s}(x+y)\right)+\psi\left(a^{s}(x-y)\right)\right\}+2\left(a+a^{2}-a^{5}-1\right) \psi\left(a^{s} x\right) \\
& \quad-10\left(a^{4}-a^{2}\right) \psi\left(\sqrt[5]{a^{s}\left(x y^{4}\right)}\right)-20\left(a^{3}-a\right) \psi\left(\sqrt[5]{a^{s}\left(x^{3} y^{2}\right)}\right)=0
\end{aligned}
$$

for $s=0,1, \ldots, t-1$. From the definition of $f$ and (3.34), we obtain that

$$
\begin{aligned}
& \mid f(a x+y)+f(a x-y)+f(x+a y)+f(x-a y) \\
& \quad-\left(a+a^{2}\right)\{f(x+y)+f(x-y)\}+2\left(a+a^{2}-a^{5}-1\right) f(x)
\end{aligned}
$$




$$
\begin{aligned}
&-10\left(a^{4}-a^{2}\right) f\left(\sqrt[5]{x y^{4}}\right)-20\left(a^{3}-a\right) f\left(\sqrt[5]{x^{3} y^{2}}\right) \mid \\
& \leq \sum_{s=0}^{\infty} \frac{1}{a^{5 s}} \mid \psi\left(a^{s}(a x+y)\right)+\psi\left(a^{s}(a x-y)\right)+\psi\left(a^{s}(x+a y)\right)+\psi\left(a^{s}(x-a y)\right) \\
&-\left(a+a^{2}\right)\left\{\psi\left(a^{s}(x+y)\right)+\psi\left(a^{s}(x-y)\right)\right\}+2\left(a+a^{2}-a^{5}-1\right) \psi\left(a^{s} x\right) \\
&- 10\left(a^{4}-a^{2}\right) \psi\left(a^{s} \sqrt[5]{x y^{4}}\right)-20\left(a^{3}-a\right) \psi\left(a^{s} \sqrt[5]{x^{3} y^{2}}\right) \mid \\
& \leq \sum_{s=t}^{\infty} \frac{1}{a^{5 s}} \mid \psi\left(a^{s}(a x+y)\right)+\psi\left(a^{s}(a x-y)\right)+\psi\left(a^{s}(x+a y)\right)+\psi\left(a^{s}(x-a y)\right) \\
&-\left(a+a^{2}\right)\left\{\psi\left(a^{s}(x+y)\right)+\psi\left(a^{s}(x-y)\right)\right\}+2\left(a+a^{2}-a^{5}-1\right) \psi\left(a^{s} x\right) \\
&-10\left(a^{4}-a^{2}\right) \psi\left(a^{s} \sqrt[5]{x y^{4}}\right)-20\left(a^{3}-a\right) \psi\left(a^{s} \sqrt[5]{x^{3} y^{2}}\right) \mid \\
& \leq \sum_{s=t}^{\infty} \frac{1}{a^{5 s}}\left(-2 a^{5}+10 a^{4}+20 a^{3}-6 a^{2}-16 a+2\right) k \\
&=\frac{\left(-2 a^{10}+10 a^{9}+20 a^{8}-6 a^{7}-16 a^{6}+2 a^{5}\right) k}{a^{5}-1} \times \frac{1}{a^{5 t}} \\
&=\frac{\left(-2 a^{15}+10 a^{14}+20 a^{13}-6 a^{12}-16 a^{11}+2 a^{10}\right) k}{a^{5}-1}\left(|x|^{5}+|y|^{5}\right) .
\end{aligned}
$$

Thus $f$ satisfies (3.32) for all $x, y \in M$ with $0<|x|^{5}+|y|^{5}<\frac{1}{a^{5}}$.

In Corollary 4 , our claim is to prove (1.1) is not stable for $p=5$. Since $f$ is continuous and bounded for all $x \in M, Q$ is continuous and bounded at the origin. In view of Corollary $4, Q(x)$ must have the form $Q(x)=t x^{5}$ for any $x$ in $M$. Thus we obtain

$$
|f(x)| \leq(\alpha+|t|) \times|x|^{5} .
$$

But $n k>\alpha+|t|, n$ is positive integer.

If $x \in\left(0, \frac{1}{a^{n-1}}\right)$, then $a^{s} x \in(0,1)$ for all $s=0,1, \ldots, n-1$. Therefore, we get

$$
f(x)=\sum_{s=0}^{\infty} \frac{\psi\left(a^{s} x\right)}{a^{5 s}} \geq \sum_{s=0}^{n-1} \frac{k\left(a^{s} x\right)^{5}}{a^{5 s}}=n k x^{5}>(\alpha+|t|) \times x^{5},
$$

which contradicts (3.35). Hence (1.1) is not stable if $p=5$ in the inequality (3.30).

\section{Stability of FUnCtional EQUation(1.2)}

In this section, we determine the generalized Hyers-Ulam stability concerning the generalized Euler-Lagrange radical sextic functional equation (1.2) in modular spaces by using fixed point theory. In this section we will not provide the proof of theorems 
and corollaries, since it is similar to that of proof of Theorems and Corollaries in section 3.

For mapping $\rho: M \rightarrow X_{\xi}$, consider

$$
\begin{aligned}
& D_{s} f(x, y):=f(a x+y)+f(a x-y)+f(x+a y)+f(x-a y) \\
& \quad-\left(a+a^{2}\right)\{f(x+y)+f(x-y)\}+2\left(a+a^{2}-a^{6}-1\right)\{f(x)+f(y)\} \\
& \quad-30\left(a^{4}-a\right)\left\{f\left(\sqrt[6]{x^{4} y^{2}}\right)+f\left(\sqrt[6]{x^{2} y^{4}}\right)\right\}
\end{aligned}
$$

for all $x, y \in M$ with $a \neq 0, \pm 1$

Theorem 5. Consider a mapping $\rho: M^{2} \rightarrow[0,+\infty)$ such that

$$
\lim _{n \rightarrow \infty} \frac{1}{a^{6 n}} a \rho\left\{a^{n} x, a^{n} y\right\}=0,
$$

and

$$
\rho\{a x, a y\} \leq a^{6} \psi \rho\{x, y\}, \forall x, y \in M,
$$

for $\psi<1$. If $f: M \rightarrow X_{\xi}$ fulfill the inequality

$$
\xi\left(D_{s} f(x, y)\right) \leq \rho(x, y),
$$

$\forall x, y \in M$. Then $S_{a}: M \rightarrow X_{\xi}$ a unique sextic mapping exists, such that

$$
\xi\left(S_{a}(x)-f(x)\right) \leq \frac{1}{2 a^{6}(1-\psi)} \rho(x, 0), \forall x \in M .
$$

Where $M$ is linear space and $X_{\xi}$ is modular space which is complete with Fatou property.

All normed space implies modular space of modular $\xi(x)=\|x\|$ gives the following Corollaries 5 and 6

Corollary 5. Assume $\rho$ is a mapping from $M^{2}$ to $[0,+\infty)$ for

$$
\lim _{n \rightarrow \infty} \frac{1}{a^{6 n}} \rho\left\{a^{n} x, a^{n} y\right\}=0,
$$

and

$$
\rho\{a x, a y\} \leq a^{6} \psi \rho\{x, y\}, \forall x, y \in M, \psi<1 .
$$

If $f: M \rightarrow X$ satisfies the condition for $X$ is Banach space

$$
\left\|D_{s} f(x, y)\right\| \leq \rho(x, y),
$$

$\forall x, y \in M$. Then a unique $S_{a}: M \rightarrow X$ sextic mapping exists, hence

$$
\left\|S_{a}(x)-f(x)\right\| \leq \frac{\rho(x, 0)}{2 a^{6}(1-\psi)},
$$

for all $x \in M$. 
Theorem 6. Assume that,

$$
\lim _{n \rightarrow \infty} \kappa^{6 n} \rho\left(\frac{x}{a^{n}}, \frac{y}{a^{n}}\right)=0,
$$

where $\rho$ is a mapping from $M^{2}$ to $[0,+\infty)$ and

$$
\rho\left(\frac{x}{a}, \frac{y}{a}\right) \leq \frac{\psi}{2 a^{6}} \rho\{x, y\}, \forall x, y \in M, \psi<1 .
$$

If $f: M \rightarrow X_{\xi}$ fulfills the inequality

$$
\xi\left(D_{s} f(x, y)\right) \leq \rho(x, y),
$$

$\forall x, y \in M$. Then a unique $S_{a}: M \rightarrow X_{\xi}$ quintic mapping exists, such that

$$
\xi\left(S_{a}(x)-f(x)\right) \leq \frac{\psi}{2 a^{6}(1-\psi)} \rho(x, 0), \forall x \in M .
$$

Corollary 6. Assume that,

$$
\lim _{n \rightarrow \infty} \sigma^{6 n} \rho\left(\frac{x}{a^{n}}, \frac{y}{a^{n}}\right)=0,
$$

where $\rho$ is a mapping from $M^{2}$ to $[0,+\infty)$ and

$$
\rho\left(\frac{x}{a}, \frac{y}{a}\right) \leq \frac{\psi}{a^{6}} \rho\{x, y\}, \forall x, y \in M, \psi<1 .
$$

If $f: M \rightarrow X$ fulfills the inequality

$$
\left\|D_{s} f(x, y)\right\| \leq \rho(x, y),
$$

$\forall x, y \in M$. Then a unique $S_{a}: M \rightarrow X$ sextic mapping exists, such that

$$
\left\|S_{a}(x)-f(x)\right\| \leq \frac{\psi}{2 a^{6}(1-\psi)} \rho(x, 0), \forall x \in M .
$$

The Hyers-Ulam and generalized Hyers-Ulam stabilities are obtain in the following corollaries using Corollaries 5 and 6 .

Corollary 7. Assume $\rho$ is a mapping from $M^{2}$ to $[0,+\infty), X$ be a Banach space and $\varepsilon \geq 0$ be a real number such that

$$
\lim _{n \rightarrow \infty} \frac{1}{a^{6 n}} \rho\left\{a^{n} x, a^{n} y\right\}=0,
$$

and

$$
\rho\{a x, a y\} \leq a^{6} \psi \rho\{x, y\}, \forall x, y \in M, \psi<1 .
$$

If $f: M \rightarrow X$ fulfills

$$
\left\|D_{s} f(x, y)\right\| \leq \varepsilon,
$$


$\forall x, y \in M$. Then a unique $S_{a}: M \rightarrow X$ sextic mapping exists and defined by $S_{a}(x)=$ $\lim _{n \rightarrow \infty} \frac{f\left(a^{n} x\right)}{a^{6 n}}$ so that

$$
\left\|S_{a}(x)-f(x)\right\| \leq \frac{\varepsilon}{2\left(a^{6}-1\right)},
$$

for all $x \in M$ and $a \neq 0, \pm 1$.

Corollary 8. If $f: M \rightarrow X$ fulfills the inequality for $M$ and $X$ are linear space and Banach space, respectively.

$$
\left\|D_{s} f(x, y)\right\| \leq \varepsilon\left(\|x\|^{p}+\|y\|^{q}\right),
$$

$\forall x, y \in M$ with $0 \leq p, q<6$ or $p, q>6$ for some $\varepsilon \geq 0$. Then a unique sextic mapping $S_{a}: M \rightarrow X$ exists and defined by $S_{a}(x)=\lim _{n \rightarrow \infty} \frac{f\left(a^{n} x\right)}{a^{6 n}}$, so that

$$
\left\|S_{a}(x)-f(x)\right\| \leq \frac{\varepsilon}{\left|2\left(a^{6}-a^{p}\right)\right|}\|x\|^{p}, \forall x \in M, a \neq 0, \pm 1 .
$$

In Corollary 8, we are providing counter example for (1.2) is not stable for $p=6$.

Example 2. If $\psi: M \rightarrow X$ fulfills the inequality for $M$ and $X$ are linear space and Banach space, respectively. Let $k \geq 0$ be a real number such that

$$
\psi(x)= \begin{cases}k x^{6}, & \text { if }|x|<1, \\ k, & \text { otherwise, }\end{cases}
$$

and a function $f: M \rightarrow X$ is defined by

$$
f(x)=\sum_{s=0}^{\infty} \frac{\varphi\left(a^{s} x\right)}{\left(a^{6}\right)^{s}}
$$

for all $x \in M$. Then $f$ satisfies the functional inequality

$$
\begin{aligned}
& \mid f(a x+y)+f(a x-y)+f(x+a y)+f(x-a y) \\
& \quad-\left(a+a^{2}\right)\{f(x+y)+f(x-y)\}+2\left(a+a^{2}-a^{6}-1\right)\{f(x)+f(y)\} \\
& \quad-30\left(a^{4}-a\right)\left\{f\left(\sqrt[6]{x^{4} y^{2}}\right)+f\left(\sqrt[6]{x^{2} y^{4}}\right)\right\} \mid \\
& \quad \leq \frac{\left(-54 a^{13}+6 a^{14}-4 a^{18}+60 a^{16}\right) k}{a^{6}-1}\left(|x|^{6}+|y|^{6}\right)
\end{aligned}
$$

for all $x, y \in M$. Then there do not exist a sextic mapping $S: M \rightarrow X$ and a constant $\alpha>0$ such that

$$
|f(x)-S(x)| \leq \alpha|x|^{6} \quad \text { for all } x \in M
$$

Proof. Now

$$
|f(x)| \leq \sum_{s=0}^{\infty} \frac{\left|\psi\left(a^{s} x\right)\right|}{\left|a^{6 s}\right|}=\sum_{s=0}^{\infty} \frac{k}{a^{6 s}}=\frac{a^{6} k}{a^{6}-1} .
$$

Therefore we see that $f$ is bounded. We are going to prove that $f$ satisfies (4.23). 
If $x=y=0$, then (4.23) is trivial. If $|x|^{6}+|y|^{6} \geq \frac{1}{a^{6}}$, then the left-hand side of (4.23) is less than $\left(\frac{-54 a^{7}+6 a^{8}-4 a^{12}+60 a^{10}}{a^{6}-1} k\right)$. Now suppose that $0<|x|^{6}+|y|^{6}<\frac{1}{a^{6}}$. Then there exists a positive integer $t$ such that

$$
\frac{1}{\left(a^{6}\right)^{t+1}} \leq|x|^{6}+|y|^{6}<\frac{1}{\left(a^{6}\right)^{t}}
$$

so that

$$
\left(a^{6}\right)^{t-1} x^{6}<\frac{1}{a^{6}},\left(a^{6}\right)^{t-1} y^{6}<\frac{1}{a^{6}}
$$

and, consequently,

$$
\begin{aligned}
a^{t-1}(x), a^{t-1}(y), a^{t-1}(a x+y), a^{t-1}(a x-y), a^{t-1}(x+a y), \\
\quad a^{t-1}(x-a y), a^{t-1}(x+y), a^{t-1}(x-y), \\
\quad a^{t-1}\left(\sqrt[6]{x^{4} y^{2}}\right), a^{t-1}\left(\sqrt[6]{x^{2} y^{4}}\right) \in(-1,1) .
\end{aligned}
$$

Therefore for each $s=0,1, \ldots, t-1$, we have

$$
\begin{array}{r}
a^{s}(x), a^{s}(y), a^{s}(a x+y), a^{s}(a x-y), a^{s}(x+a y), a^{s}(x-a y), a^{s}(x+y), \\
a^{s}(x-y), a^{s}\left(\sqrt[6]{x^{4} y^{2}}\right), a^{s}\left(\sqrt[6]{x^{2} y^{4}}\right) \in(-1,1) .
\end{array}
$$

and

$$
\begin{aligned}
& \psi\left(a^{s}(a x+y)\right)+\psi\left(a^{s}(a x-y)\right)+\psi\left(a^{s}(x+a y)\right)+\psi\left(a^{s}(x-a y)\right) \\
& \quad-\left(a+a^{2}\right)\left\{\psi\left(a^{s}(x+y)\right)+\psi\left(a^{s}(x-y)\right)\right\} \\
& \quad+2\left(a+a^{2}-a^{6}-1\right) \psi\left(a^{s} x+a^{s} y\right) \\
& \quad-30\left(a^{4}-a\right)\left\{\psi\left(a^{s} \sqrt[6]{x^{4} y^{2}}\right)+\psi\left(a^{s} \sqrt[6]{x^{2} y^{4}}\right)\right\}=0
\end{aligned}
$$

for $s=0,1, \ldots, t-1$. From the definition of $f$ and (3.34), we obtain that

$$
\begin{aligned}
& \mid f(a x+y)+f(a x-y)+f(x+a y)+f(x-a y) \\
& \quad-\left(a+a^{2}\right)\{f(x+y)+f(x-y)\} \\
& \quad+2\left(a+a^{2}-a^{6}-1\right)\{f(x)+f(y)\} \\
& \quad-30\left(a^{4}-a\right)\left\{f\left(\sqrt[6]{x^{4} y^{2}}\right)+f\left(\sqrt[6]{x^{2} y^{4}}\right)\right\} \mid \\
& \left.\leq \sum_{s=0}^{\infty} \frac{1}{a^{6 s}} \mid \psi\left(a^{s}(a x+y)\right)+\psi\left(a^{s}(a x-y)\right)+\psi\left(a^{s}(x+a y)\right)\right) \\
& \quad+\psi\left(a^{s}(x-a y)-\left(a+a^{2}\right)\left\{\psi\left(a^{s}(x+y)\right)+\psi\left(a^{s}(x-y)\right)\right\}\right. \\
& \quad+2\left(a+a^{2}-a^{6}-1\right) \psi\left(a^{s} x+a^{s} y\right) \\
& \quad-30\left(a^{4}-a\right)\left\{\psi\left(a^{s} \sqrt[6]{x^{4} y^{2}}\right)+\psi\left(a^{s} \sqrt[6]{x^{2} y^{4}}\right)\right\} \mid
\end{aligned}
$$




$$
\begin{aligned}
\leq & \sum_{s=t}^{\infty} \frac{1}{a^{6 s}} \mid \psi\left(a^{s}(a x+y)\right)+\psi\left(a^{s}(a x-y)\right)+\psi\left(a^{s}(x+a y)\right) \\
& +\psi\left(a^{s}(x-a y)\right)-\left(a+a^{2}\right)\left\{\psi\left(a^{s}(x+y)\right)+\psi\left(a^{s}(x-y)\right)\right\} \\
& +2\left(a+a^{2}-a^{6}-1\right) \psi\left(a^{s} x+a^{s} y\right) \\
& -30\left(a^{4}-a\right)\left\{\psi\left(a^{s} \sqrt[6]{x^{4} y^{2}}\right)+\psi\left(a^{s} \sqrt[6]{x^{2} y^{4}}\right) \mid\right. \\
\leq & \sum_{s=t}^{\infty} \frac{1}{a^{6 s}}\left(-54 a+6 a^{2}-4 a^{6}+60 a^{4}\right) k \\
= & \frac{\left(-54 a^{7}+6 a^{8}-4 a^{12}+60 a^{10}\right) k}{a^{6}-1} \times \frac{1}{a^{6 t}} \\
= & \frac{\left(-54 a^{13}+6 a^{14}-4 a^{18}+60 a^{16}\right) k}{a^{6}-1}\left(|x|^{6}+|y|^{6}\right) .
\end{aligned}
$$

Thus $f$ satisfies (4.23) for all $x, y \in M$ with $0<|x|^{6}+|y|^{6}<\frac{1}{a^{6}}$.

The remaining part of proof is similar to that of previous Example 1.

\section{CONCLUSiON}

Mainly, we introduced new generalized Euler-Lagrange radical sextic and quintic functional equations with their general solution and investigated their generalized Hyers-Ulam stability in modular spaces by using fixed point theory with suitable counter examples.

\section{REFERENCES}

[1] T. Aoki, "On the stability of the linear transformation in Banach spaces." J. Math . Soc.Japan, vol. 2, pp. 64-66, 1950.

[2] A. Bodaghi, P. Narasimman, K. Ravi, and B. Shojaee, "Mixed type of additive and quintic functional equations." Annales Mathematicae Silesianae, vol. 29, pp. 35-50, 2015.

[3] I. Cho, D. Kang, and H. Koh, "Stability problems of quintic mappings in quasi- $\beta$-normed spaces." J. Inequal. Appl., no. Art. ID 368981, p. 9 pp., 2010.

[4] I. El-Fassi and S. Kabbaj, "On the generalized orthogonal stability of mixed type additive-cubic functional equations in modular spaces." Tbilisi Mathematical Journal, vol. 9, no. 1, pp. 231-243, 2016.

[5] Z. Eskandani and J. M. Rassias, "Stability of general $A$-cubic functional equations in modular spaces." RACSAM, no. DOI 10.1007/s13398-017-0388-5, 2017.

[6] M. B. Ghaemi, H. Majani, and M. E. Gordji, "Approximately quintic and sextic mappings on the probabilistic normed spaces.” Bull. Korean Math. Soc., vol. 49, no. 2, pp. 339-352, 2012.

[7] P. Găvruta, "A generalization of the Hyers-Ulam-Rassias stability of approximately additive mappings." J. Math. Anal. Appl., vol. 184, pp. 431-436, 1994.

[8] D. H. Hyers, "On the stability of the linear functional equation." Proc. Natl. Acad. Sci., vol. 27, pp. 222-224, 1941.

[9] P. Narasimman, J. Rassias, and K. Ravi, " $n$-dimensional quintic and sextic functional equations and their stabilities in Felbin type spaces." Georgian Math. J., vol. 23, no. 1, pp. 121-137, 2016. 
[10] J. Rassias, “On approximately of approximately linear mappings by linear mappings.” J. Funct. Anal. USA, vol. 46, pp. 126-130, 1982.

[11] T. M. Rassias, "On the stability of the linear mapping in Banacb spaces." Proc. Amer. Math. Soc., vol. 72, pp. 297-300, 1978.

[12] K. Ravi, M. Arunkumar, and J. Rassias, "On the Ulam stability for the orthogonally general Euler-Lagrange type functional equation." International Journal of Mathematical Sciences, vol. 3 , no. 08 , pp. $36-47,2008$.

[13] S. Ulam, A Colloection of the Mathematical Problems. New York: Interscience Publ., 1960.

[14] K. Wongkum, P. Kumam, Y. Cho, P. Thounthong, and P. Chaipunya, "On the generalized UlamHyers-Rassias stability for quartic functional equation in modular spaces." J. Nonlinear Sci. Appl., vol. 10, pp. 1399-1406, 2017.

[15] T. Z. Xu, J. M. Rassias, M. J. Rassias, and W. X. Xu, "A fixed point approach to the stability of quintic and sextic functional equations in quasi- $\beta$-normed spaces.” J. Inequal. Appl., no. Art. ID 423231, p. 23, 2010.

Authors' addresses

Murali Ramdoss

PG and Research Department of Mathematics, Sacred Heart College (Autonomous), Tirupattur-635 601, TamilNadu, India

E-mail address: shcrmuralieyahoo.co.in

\section{Divyakumari Pachaiyappan}

PG and Research Department of Mathematics, Sacred Heart College (Autonomous), Tirupattur-635 601, TamilNadu, India

E-mail address: divyakumarishcegmail.com

\section{Hemen Dutta}

Department of Mathematics, Gauhati University, Guwahati-781014, Assam, India

E-mail address: hemen_dutta08@rediffmail.com 